\title{
La ciudad sin Urbanismo: instalación residencial junto a secciones andinas inestables, dunas activas, bancos de arena y rodados fluviales.
}

Francisco Ferrando A.

\section{Filiación}

Geógrafo - Hidrogeomorfólogo, Director de la Escuela de Geografía de la Facultad de Arquitectura y Urbanismo de la Universidad de Chile.

\section{Palabras Claves}

Sustentabilidad urbana, zonas de riesgo, planificación urbana.

\section{Resumen}

La negligencia recurrente en el acto de instalación humana en diversos puntos del territorio, obliga a reiterar la necesidad de cautelar la sustentabilidad ambiental de la obra arquitectónica urbana. La Quebrada de San Ramón presenta condiciones de inestabilidad en su sección andina, las que se evidencian tanto por aspectos prehistóricos como históricos, así como por el estado de sus componentes físicas, lo cual configura un escenario de riesgo potencial de aluviones. Dicha situación es un antecedente insoslayable frente a proyectos de densificación del poblamiento en el sector inmediato aguas abajo del punto en que ésta quebrada abandona el marco andino. En el caso de los campos de dunas activas en el litoral chileno, algunos de gran valor científico, diversos emprendimientos "urbanísticos" están ejerciendo fuertes presiones y usos que rayan en la irracionalidad y generan en el corto y mediano plazo riego para la población y fuertes impactos socioeconómicos. Finalmente, constatamos en Temuco, a menos de un metro por sobre el nivel medio de las aguas del río Cautín, población de viviendas económicas. Se observa problemas por sedimento suelto no consolidado, riesgo de inundaciones y erosión (pérdida de "suelos de fundación" y falla de las bases y la estructura), alta humedad del suelo por napa friática a pocos centímetros de la superficie (revenimiento de pisos y paredes, problemas de salud, deterioro de muebles, etc.). Corresponde preguntarse en qué aspectos estas vecindades y sus viviendas son consecuentes con los logros contemporáneos para ofrecer marcos de vida satisfactorios.

\begin{abstract}
Urban and architectural works have to take into consideration the environmental sustainability, that has been neglected for a long time in the settlement of new urban areas.
\end{abstract}

\section{Key words}

Urban sustainability, risk zones, urban planning. 
http://revistaurbanismo.uchile.cl

\section{Sumario}

1.-Inestabilidad de la sección andina de la quebrada de Ramón y riesgo de la densificación del poblamiento en el piedmont metropolitano de Santiago

2.-Construcción sobre dunas activas, en Iquique.

3.- Construcción sobre bancos de arena y rodados fluviales de la terraza baja del río Cautín, Temuco

\section{1.- Inestabilidad de la sección andina de la quebrada de Ramón y riesgo de la densificación del poblamiento en el piedmont metropolitano de Santiago}

Con motivo de la realización de dos proyectos binacionales IPGH-OEA sobre asentamientos urbanos en áreas de riesgo aluvional y de remoción en masa, a saber, Santiago - Mendoza y Santiago - Río de Janeiro, se ha recorrido la parte alta de la Quebrada de Ramón en dos oportunidades en período reciente, en conjunto con especialistas de los países involucrados, contando para ello con la autorización y apoyo de CONAF y EMOS.

Este reconocimiento ha permitido evaluar el estado de laderas y depósitos sedimentarios antiguos y actuales, y de la vegetación, así como de los procesos en actividad y potenciales que pueden afectar este medio y proyectarse sobre la parte alta de la ciudad inmediata al punto donde la quebrada abandona el marco montañoso preandino.

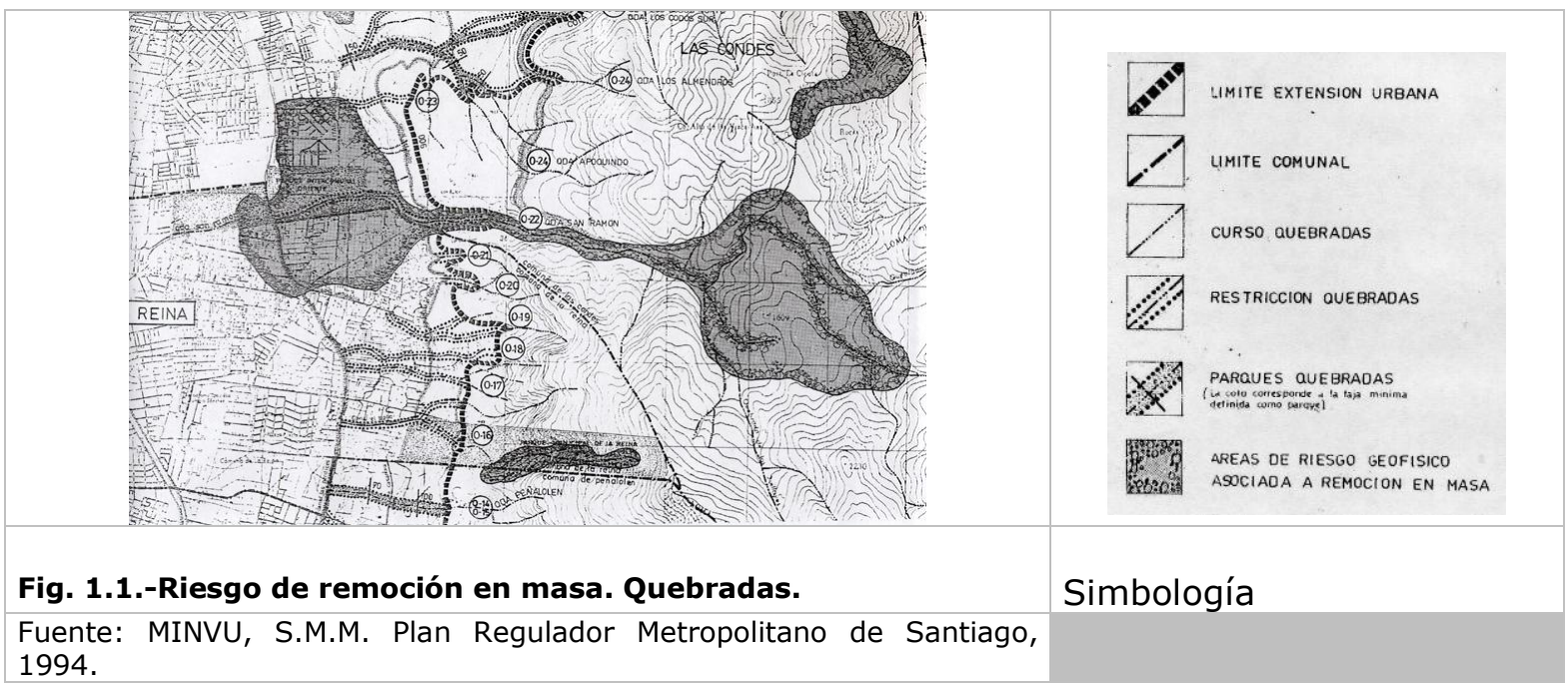




\section{Características Generales de la Cuenca}

La Quebrada de Ramón está constituida por una hoya interior de forma ovalada que comprende la mayor parte de su superficie andina (Zona de Producción), la que se continúa en un estrecho corredor en forma de cañón el cual se orienta en sentido $\mathrm{E}-\mathrm{W}$ (Zona de Transferencia). La superficie de estos sectores alcanza los 36,26 Kmts², y las alturas oscilan entre 800 m.s.n.m. (punto de salida al piedmont) y 3.249 m.s.n.m. (Cerro San Ramón).

La pendiente media de la sección andina de la cuenca alcanza porcentajes de hasta $30 \%\left(13.5^{\circ}\right)$, registrándose en la sección media y superior de las laderas valores que llegan a superar la pendiente de equilibrio de los taludes $\left(32^{\circ}\right)$. Las precipitaciones medias se incrementan con la altura de $450 \mathrm{~mm}$ a los $850 \mathrm{~m}$ a $900 \mathrm{~mm}$ a los $2000 \mathrm{~m}$, pasando luego a precipitaciones sólidas (López, 1996).

La hoya interior está configurada por una serie de quebradas afluentes que se disponen en forma radial y que drenan sectores montañosos de fuertes pendientes y constitución geológica sedimentario-volcánica, la que se encuentra fuertemente alterada tanto física como químicamente producto de haber sido sometida a intensos plegamientos, fallamientos e intrusiones graníticas acompañadas de manifestaciones hidrotermales locales.

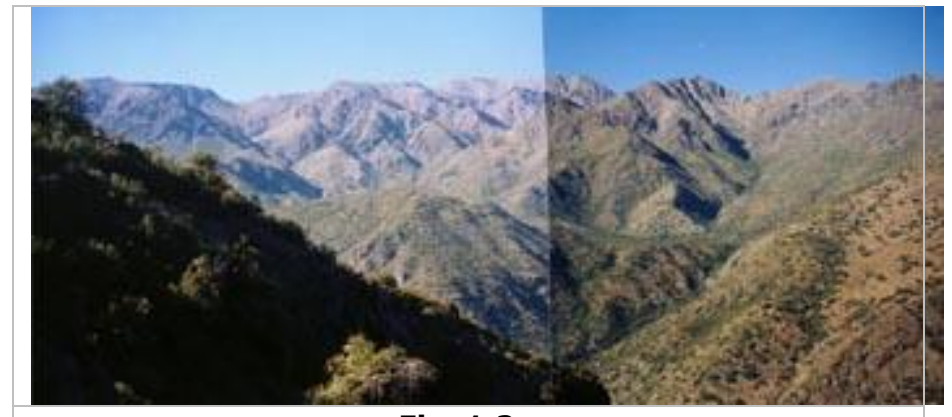

Fig. 1.2.-

Vegetacionalmente, la superficie de la cuenca se divide en $2,81 \mathrm{~km}^{2}$ de matorral arbustivo denso; $14,45 \mathrm{~km}^{2}$ de matorral arbustivo ralo; $8,6 \mathrm{~km}^{2}$ de herbáceas de altura; y $10,67 \mathrm{~km}^{2}$ de suelo desnudo (López, op. cit.), lo cual habla de una baja protección y estabilización del suelo y los mantos detríticos en gran parte de la superficie de la cuenca.

Por su parte, el cañón de la sección inferior se presenta fuertemente encajado en rocas de similar naturaleza y estado, así como cortando antiguas remociones en masa que han edificado relieves pedemontanos de forma acolinada con alturas máximas de 1000 $\mathrm{m}$, cota alcanzada en la ribera $\mathrm{N}$. Estos relieves dan paso inmediato a los conos torrenciales edificados a merced de los relieves señalados y sus aportes de materiales. 


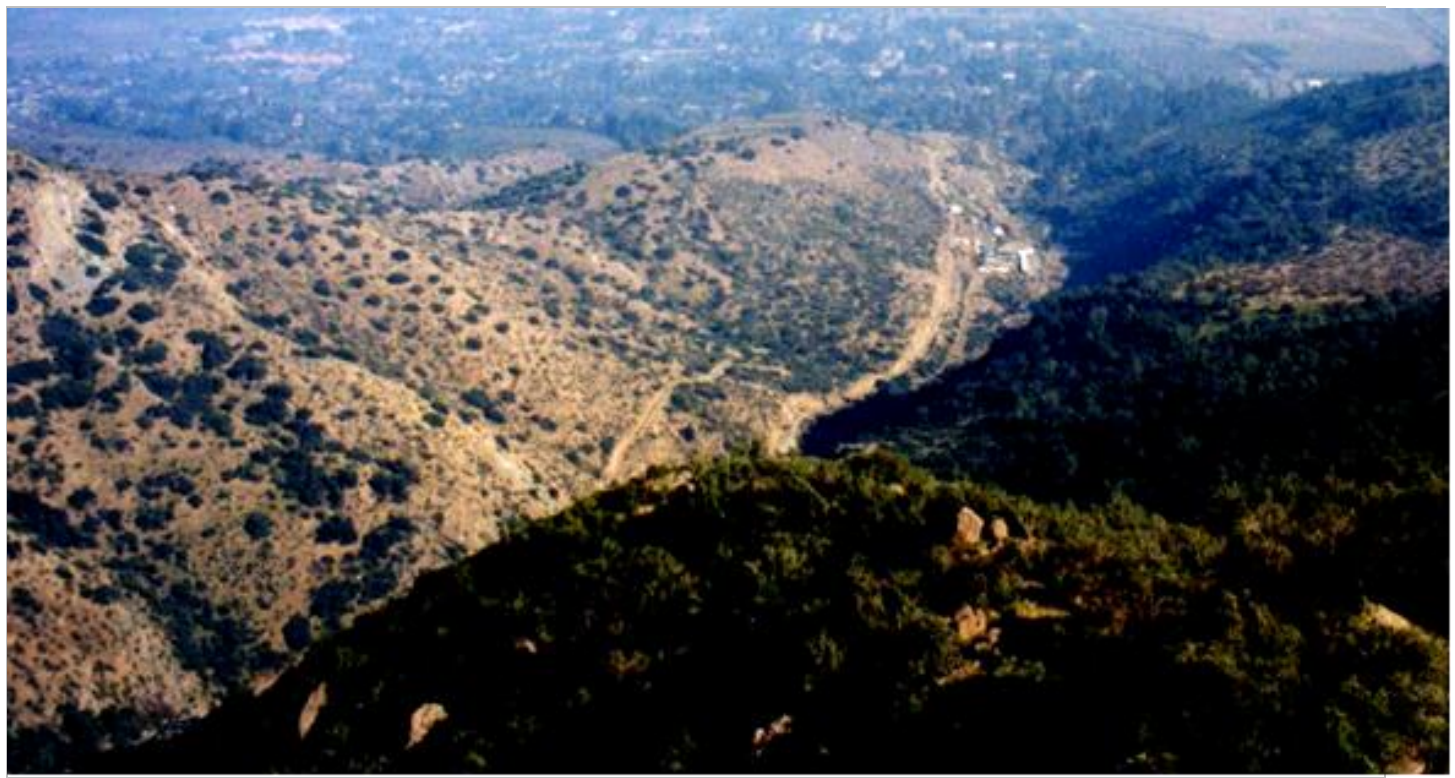

Figs. 1.3.- a y b

Hacia aguas abajo, ya en el área urbanizada, el cauce presenta intervenciones como enrocado lateral y una serie de muretes transversales destinados a frenar la velocidad de escurrimiento de las aguas y la parte de la carga detrítica fina entre el recinto de CONAF y la calle Padre Hurtado. En este punto existe un canal transversal de agua y un puente con escasa luz, obras que potencialmente pueden obstruir el paso de las aguas u otro tipo de flujo por acumulación de material detrítico (bloques de roca, ramas, etc.).

\section{Estado de equilibrio morfológico}

En la hoya interior cabe destacar una serie de situaciones que indican una alta inestabilidad, partiendo por la existencia de voluminosos depósitos de remoción en masa abortados o remanentes de aquel proceso que llevó los depósitos de igual origen existentes a la salida de la precordillera hacia el W. (Ferrando y Mikkan, 2000). A ellos se suma una gran cantidad de materiales coluviales, deslizamientos de laderas y derrumbres de frentes rocosos, lo cual está revelando la alta producción actual de materiales detríticos y su aporte a los numerosos cauces y sus respectivas corrientes hídricas, vehículos de concentración y transporte de estos hacia las partes bajas con diversos grados de intensidad, según sean los aportes pluviales y/o de aguas de fusión nival.

Los cursos de agua referidos, no sólo están transportando los materiales aportados por las laderas directamente al cauce, sino también están retomando carga de los materiales de remoción en masa interiores, para lo cual han disectado profundamente estos últimos, socavado lateralmente y generado escarpes casi verticales de más de 
http://revistaurbanismo.uchile.cl

20 metros de desnivel, los que están sufriendo permanentes derrumbes y retroceso de sus frentes. Dichos sedimentos han estado siendo aportados directamente a los cauces, en los cuales se han ido acumulando por falta de competencia de los cursos de agua, dado su escaso caudal promedio.

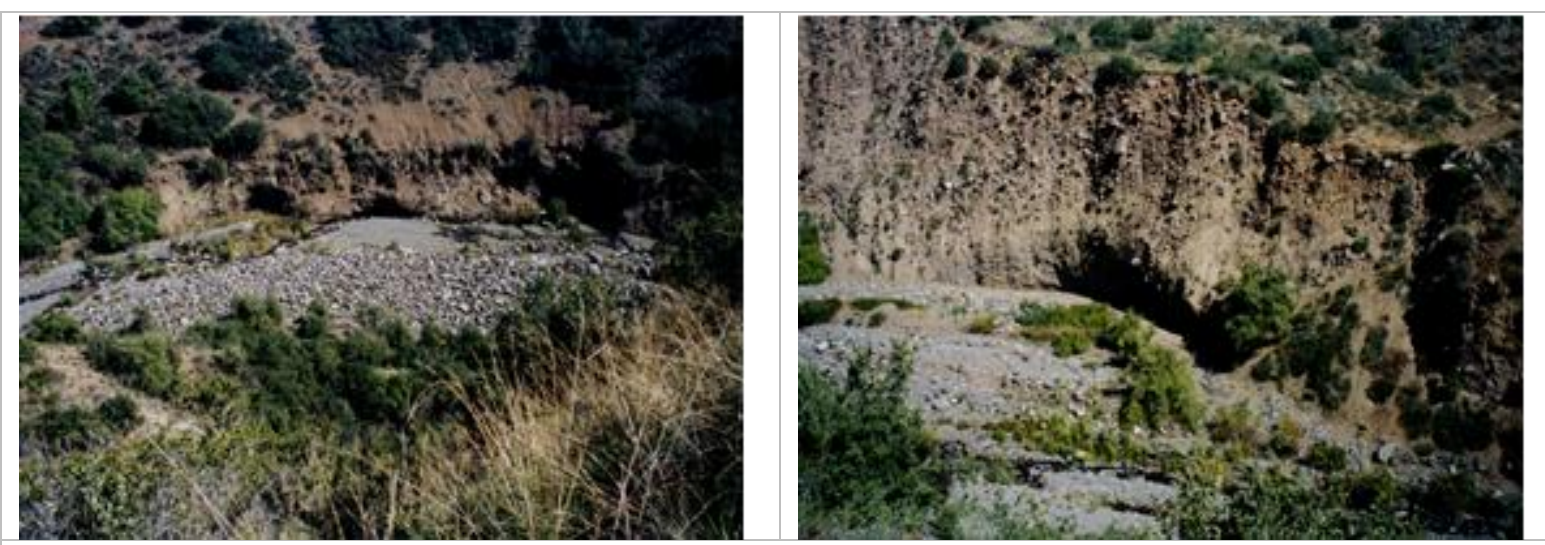

Figs. 1.4.- y 1.5.-

De igual modo, los flancos del cañón, en cuyo trayecto se produce la máxima concentración de energía y masa proveniente de la cuenca alta, están sufriendo derrumbes y deslizamientos locales, tanto producto de la evolución natural de los relieves, en parte asistidos por actividad sísmica, como de procesos inducidos por la intervención antrópica (Ej.: Camino de EMOS por la vertiente S.). Producto de lo anterior, en esta sección de la quebrada existe también una gran cantidad de depósitos de granulometría mayor (grandes bloques de roca) y media, los cuales están a disposición de potenciales flujos barrosos originables en las partes altas.

Cabe mencionar como paliativo, que se ha estado produciendo una importante recuperación de la vegetación nativa, principalmente del matorral arbustivo, dado el estatus legal actual de la cuenca, lo que permite establecer un incremento en la estabilidad de los materiales detríticos superficiales. Sin embargo, este rol de la vegetación se ve restringido en relación con desestabilizaciones más profundas, pérdidas de estabilidad de espesos depósitos sedimentarios o derrumbes de rocas de volumen mayor. Por ello es común observar acumulaciones de troncos y ramas en algunos cauces, evidencia de su incorporación a los materiales trasladados por aluviones o remociones.

Si se suman los antecedentes expuestos en cuanto a las características constitutivas de la cuenca, los ingentes volúmenes de detritos que forman parte de remociones antiguas, los depósitos de reciente generación y aquellos que resultan de procesos actuales, con las fuertes pendientes de una cuenca alta de gran compacidad y alta torrencialidad, más los depósitos de bloques y aluvionales remanentes existentes en el cañón de la parte baja, es evidente que nos encontramos frente a una cuenca pedemontana de alta potencialidad a generar remociones en masa, es decir, flujos de barro y bloques de considerable densidad, los que no respetan cauces ni defensas 
http://revistaurbanismo.uchile.cl

como las actualmente existentes en la parte inmediata aguas abajo del sector de EMOS y CONAF.

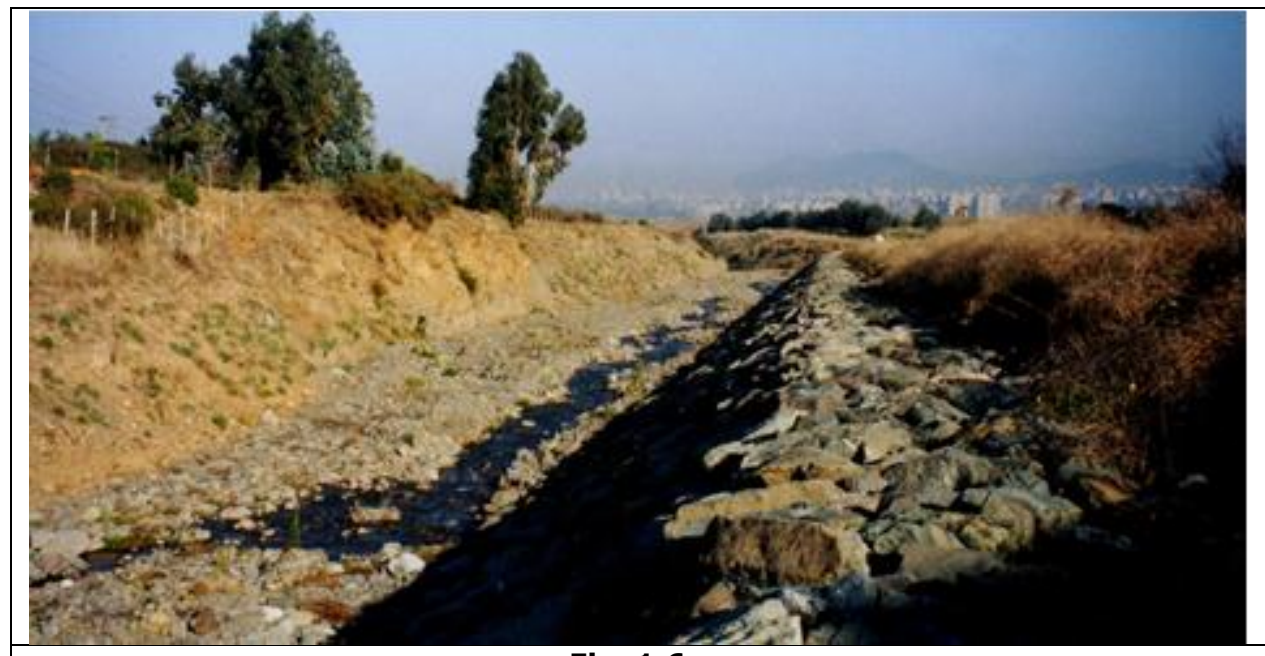

Fig. 1.6.-

Tampoco un flujo de estas características (Debris Flow) respeta la sinuosidad de los cursos de agua o cauces menores, sino que sigue la alineación principal del cauce episódico si este es encajado (cañón), sin doblar como si fuese sólo agua con una proporción menor de carga de sedimentos.

Debe tenerse presente que no se está hablando de potencialidad de crecidas decenales o cincuentenarias solamente, sino de la existencia de condiciones favorables a la generación de flujos mucho más densos, de un momentum mucho mayor y que avanzan como una ola de barro y piedras de varios metros de altura con un incontenible poder arrasador. Aún más, la llegada de un flujo de esta naturaleza, lo cual no es ajeno a la historia de esta quebrada, se encontraría a su salida a la zona alta del piedmont con un pequeño embalse para riego, el cual le proporcionaría el elemento líquido necesario para potenciar aún más su proyección areal y su poder destructor.

\section{Relación entre precipitaciones y umbral aluvional}

Como resultado de los estudios realizados por Hausser (1985) en el sector preandino de Santiago, se establece una relación genética entre el monto de las precipitaciones por día y el desencadenamiento de remociones en masa, llegándose a que se configuran escenarios de alta probabilidad de ocurrencia de estos procesos si la lluvia supera los $60 \mathrm{~mm}$ en 24 horas. Cabe anotar que recientemente, el día 30 de julio/2001 se registró $50 \mathrm{~mm}$ en $24 \mathrm{hrs}$. y que el mapa sinóptico indicaba la entrada de dos nuevos frentes de similares características. 
http://revistaurbanismo.uchile.cl

Por su parte, López (op. cit.) realizó análisis estadísticos de los registros pluviométricos de la estación meteorológica de Cerro Calán obteniendo como resultado que el período de retorno de tal situación es de 3 años, con una probabilidad de excedencia del $30 \%$. De acuerdo a datos de D.G.A., en el período 1980-1995 precipitaciones iguales o superiores a 60mm / 24 hrs. se registraron en los años 1981, 1984, 1986, 1987 (89mm/24hrs), 1991 y 1992. Si bien en los momentos correspondientes de dichos años se registraron algunas remociones locales, además de crecidas, desbordes e inundaciones, no hubo desencadenamiento de flujos aluvionales semejantes al ocurrido en mayo de 1993, donde las precipitaciones registradas en la estación de cerro Calán sólo alcanzaron a 30,9 mm/24 hrs., y a una intensidad máxima de 9,8 mm/hora. (R.E.G., 1993).

Por lo tanto, cabe señalar que existen otras condiciones meteorológicas, tanto previas como del momento, que pueden conducir a la generación de estos procesos, y no sólo altos montos de precipitación como los señalados. De hecho, cambios bruscos en la estructura térmica vertical de la atmósfera, como es el ascenso de la isoterma de $0^{\circ} \mathrm{C}$. que marca el límite entre precipitación líquida (lluvia) y sólida (nieve) tiene una fuerte influencia en la génesis de flujos detrítico lodosos, como en el caso del aluvión de mayo 1993.

Como conclusión de estudios llevados a cabo por Ferrando y Mikkan (op. cit.) en diversas cuencas del piedmont de Santiago y de Mendoza, se estableció que:

- Cada evento aluvional es el resultado de una combinación aleatoria única y específica de factores tanto estables (constitutivos del medio) como variables, siendo estos últimos los asociados al clima local (mecanismos detonantes).

- El incremento de la predisponibilidad de los componentes del medio físico a perder parte de su masa, está directamente relacionado con la producción y acumulación progresiva de materiales detríticos en cubiertas o depósitos no consolidados, 0 inestables en la zona de producción de las cuencas.

- En este sentido, y considerando la manifestación de los mecanismos detonantes en el tiempo como algo propio del azar climático, aunque con mayor probabilidad en ciertas estaciones, la ocurrencia, o no ocurrencia, de este tipo de procesos en cada una de las cuencas estudiadas, o el lapso de tiempo transcurrido, es inversamente proporcional a la potencialidad de generación de un nuevo o primer evento aluvional.

- Lo anterior se basa en el hecho que, por una parte, todas las cuencas estudiadas presentan en su superficie características constitutivas específicas que generan escenarios proclives a sufrir y potenciar estos procesos $y$, por otra parte, el hecho que aquellas cuencas que en el pasado reciente han sufrido evacuación de sus cubiertas detríticas, por lo general en forma parcial, pueden ser consideradas como con menor potencialidad de una nueva manifestación en el corto plazo frente a aquellas que, en similares condiciones, no han sido objeto de estos mecanismos, manteniendo e incrementando la masa detrítica disponible en las partes altas. 
http://revistaurbanismo.uchile.cl

- Todo lo expuesto hace ver lo difícil de la predicción en el corto plazo, aunque si es posible indicar aquellas cuencas que representan una mayor amenaza potencial, es decir, condiciones intrínsecas propicias a las remociones en masa, para que las medidas de manejo preventivo puedan ser planificadas y efectuadas con tiempo.

De acuerdo a lo señalado previamente, y a lo establecido por López (op. cit.) en cuanto a la desnudez del suelo por sobre los $2000 \mathrm{~m}$ de altura, el alto potencial erosivo de los sistemas hidrológicos, abundantes sectores generadores de material detrítico y presencia de sedimentos no consolidados en las vertientes, así como a la existencia de importantes volúmenes de depósitos de remociones en masa antiguas en tanto fuentes aportantes de carga sólida a los cursos de agua (Ferrando y Mikkan, op. cit.), queda claro que existen las condiciones propicias para la generación de mecanismos aluvionales, requiriéndose sólo el concurso de las condiciones desencadenantes.

Estas, según Peña y Vidal (1993), tienen relación directa con la acumulación de nieve por sobre los $1800 \mathrm{~m}$ de altura, y con la penetración de frentes de precipitación cálidos que eleven rápidamente la isoterma de $0^{\circ} \mathrm{C}$., lo que en términos prácticos significa que el área de producción hídrica y aportante de escurrimiento se eleva por sobre el $100 \%$ de la superficie de la cuenca (López, op. cit.). Esto se explica porque en los sectores altos (sobre $1800 \mathrm{~m}$ ) se produce una sumatoria del agua de fusión nival con el agua de lluvia en forma simultánea.

\section{Poblamiento del Piedmont de la Quebrada San Ramón 1955 - 1995}

La zona del piedmont de la Quebrada de Ramón en el año 1955 se distribuía en pradera natural y propiedad mediana en la parte norte, y de granjas en la parte sur. La densidad del poblamiento era muy baja.

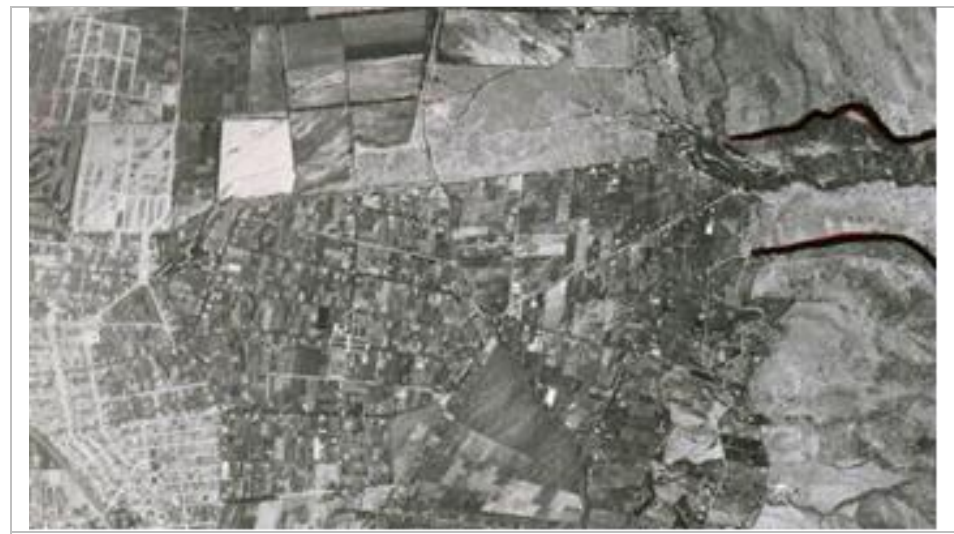

Fig. 1.7.- 
http://revistaurbanismo.uchile.cl

Ya en 1995, la situación era completamente diferente, y se registran cambios de uso de rural a urbano junto con una notoria densificación del poblamiento, predominando casas quinta y mini parcelas al sur del eje de la quebrada, las que en la Fig. 7, se indican como "parcelas de agrado". Hacia el sector norte del cauce también se observa cambios de importancia, registrándose la instalación del Centro Nuclear, áreas deportivas y sectores urbanizados de alta densidad. Cabe recordar que los sectores tanto al norte como al sur de la quebrada fueron afectados por el flujo de lodo de mayo de 1993.

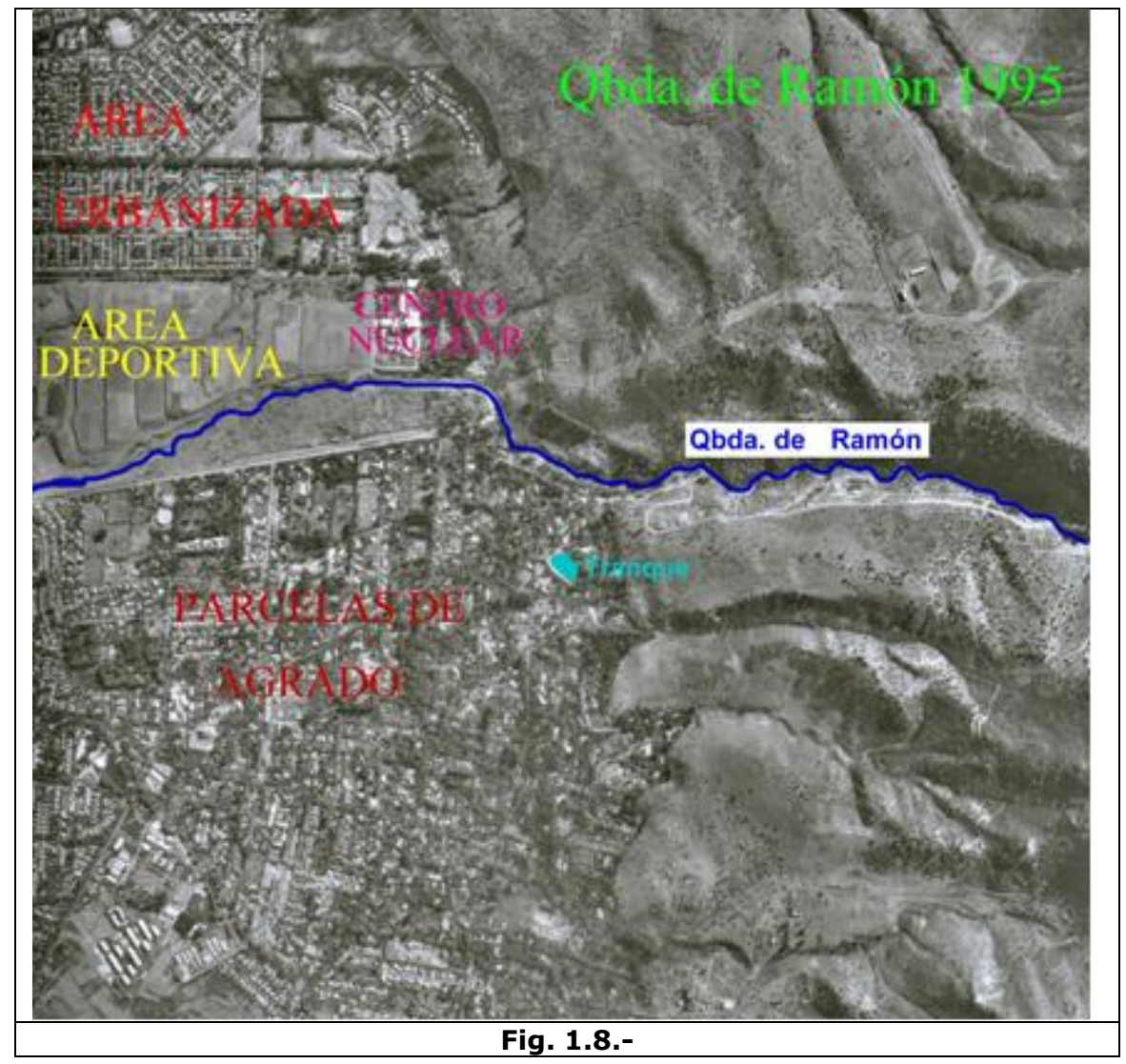

Se ha realzado la ubicación de un tranque para riego existente inmediatamente al sur de la salida de la quebrada al piedmont, sector que corresponde al ápice del cono de la quebrada, el cual presenta una composición y estructura que evidencia comportamientos torrenciales en su fase de construcción y evolución. Este tranque podría verse afectado e incorporar su masa hídrica al flujo en caso de una eventual remoción en masa de gran magnitud, potenciando su proyección y poder de destrucción de la misma. 
http://revistaurbanismo.uchile.cl

Al interior, en el sector del cauce encajonado se visualizan las instalaciones de E.M.O.S., las cuales debieron ser reconstruidas en parte con posterioridad al aluvión de mayo/99.

\section{Riesgo de instalaciones y de la densificación del poblamiento}

Es en base de estos antecedentes que se puede concluir que, además del alto riesgo que corren las instalaciones existentes en el sector del cañón, inmediatamente aguas abajo existe un triángulo de alto riesgo que tiene su vértice a la salida de la quebrada al piedmont, abriéndose a partir de este punto en un abanico que va de WSW (Calle Onofre Jarpa) a WNW (hacia calle Nueva Bilbao), involucrando en ello sectores ubicados tanto y principalmente al S. como al N. de la calle Valenzuela Puelma. Lo anterior debido a los voluminosos depósitos de escombros con que se han elevado y nivelado escalonadamente los terrenos al Norte del eje del cauce de la Quebrada San Ramón y oriente de la Av. Padre Hurtado.

Dado lo anterior, y sin entrar a referirse a aluviones lodosos y de menores consecuencias como el que en mayo 1993 afectó a esta quebrada, se puede estimar de alto riesgo el incremento de la densidad de poblamiento en dichos sectores y, más bien, se recomienda una disminución de esta y la creación de áreas de reserva y protección con usos que no entrañen la permanencia estable de personas ni instalaciones de cuidado por las materias primas o procesos que desarrollen (Ej.: Recreación).

Si, un aluvión menor como el de 1993 destruyó la bocatoma de EMOS, amén de instalaciones de protección del cauce en la parte alta de la cuenca como se observa en las fotos siguientes, una remoción en masa destruiría la totalidad de ellas.

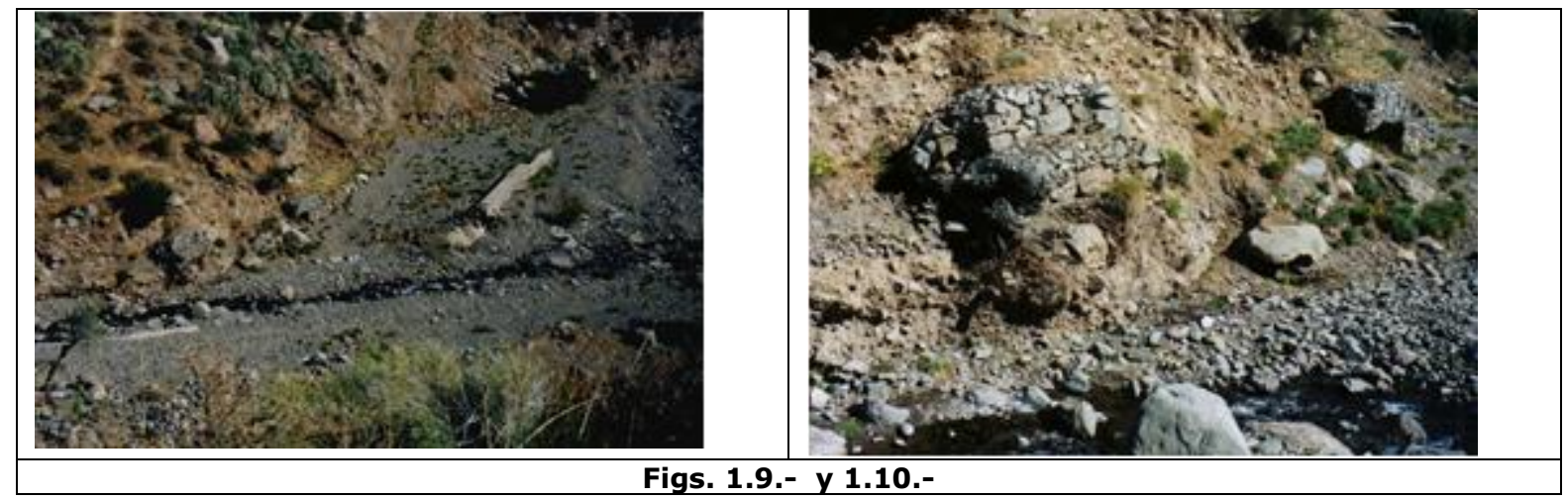




\section{Recomendaciones}

Si bien los cálculos de probabilidad que se han efectuado indicarían una baja a muy baja recurrencia de remociones en masa de gran magnitud en esta cuenca, cabe mencionar, por una parte, que hace mucho tiempo que esto no ocurre y, por otra, que la naturaleza tiene un comportamiento aleatorio, ante lo cual las predicciones no tienen más valor que la de establecer un escenario de riesgo potencial, siendo lo más adecuado y económico adoptar las medidas de prevención pertinentes, que van desde un manejo integral de la cuenca alta y media para estabilización de laderas y control de torrentes en base de medidas estructurales y biológicas, y definición de áreas de preservación, hasta recomendaciones de restricción a la densificación del poblamiento.

Es propio respetar los cauces de la naturaleza (los caminos del agua) y ofrecerle los espacios necesarios para la disipación de sus procesos y energía procurando el menor daño y costo social posible, sobre todo cuando se trata de aquellos mecanismos en los que el hombre sólo puede intervenir restringidamente y con efectos paliativos o de mitigación de las posibles consecuencias.

El tratamiento adecuado de la cuenca alta implica atacar el problema desde sus orígenes y no cuando este ya se encuentra desencadenado y con toda su energía, con el objetivo de poder regular en parte la concentración y disipación de esta, difiriéndola en el tiempo.

Paralelamente, todo tipo de acción que se haga en este sentido conducirá a la reducción de la posibilidad de ocurrencia de procesos aluvionales así como a la disminución de su magnitud.

\section{2.-Construcción sobre dunas activas, en Iquique.}

\section{Introducción}

Chile es un país vulnerable, suma de angostura y pendientes, de sismicidad y volcanismo, de cuencas y torrencialidad, de alturas y pluviosidad, de aluviones y movimientos en masa.

Históricamente, y en este contexto, la mayoría de las ciudades de Chile han sido fundadas a orillas de ríos, así como en terrenos inestables, locaciones calificadas como áreas de alto riesgo. Estos hechos tal vez podrían justificarse por la falta de conocimientos que antes existía sobre la dinámica del medio geográfico físico. Pero, que en los últimos años, la expansión de las áreas urbanas se siga realizando hacia sectores que extrañan una alta peligrosidad, no tiene calificativo.

Como casos de ciudades que han presentado problemas, se puede mencionar: Arica (crecidas del río San José); Antofagasta (activamiento de quebrada y aluvión); San Antonio (activamiento de estero y flujo de barro); El Quisco (construcción en dunas y 
http://revistaurbanismo.uchile.cl

cordones litorales entre el mar y marismas); Concepción (inundación de poblaciones construidas en sectores bajos a orillas del río Bío-Bío); Punta Arenas (aluvión del río Las Minas).

En relación con proyectos urbanos en desarrollo, se advierte la generación de problemas en los suelos de fundación del conjunto de edificios del complejo turístico de la Avenida del Mar, en La Serena, debido a la edificación en altura sobre una terraza marina con cementación calcárea y nivel friático alto. También se puede mencionar el proceso de urbanización de sectores de dunas semiestabilizadas sobre el borde del acantilado costero en el camino Concón-Reñaca.

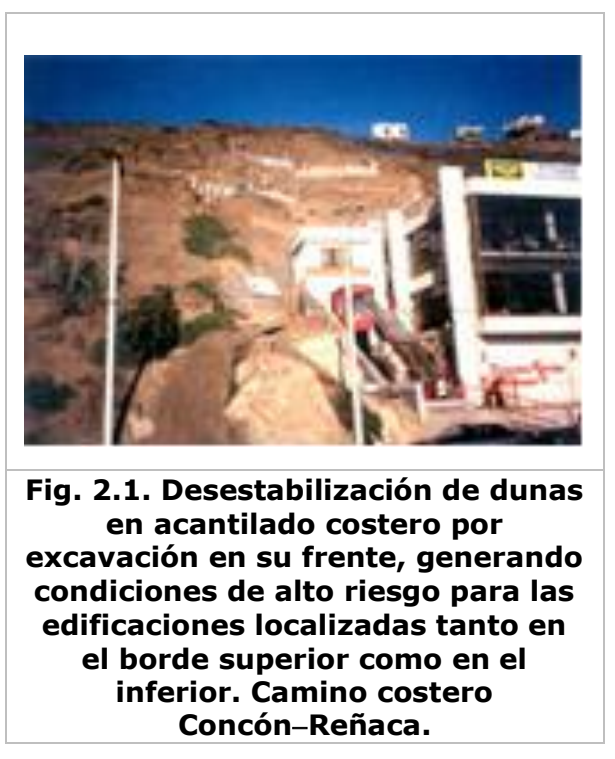

Entre las acciones y situaciones más frecuentes emprendidas por el hombre y que generan situaciones de riesgo se cuentan: relleno de quebradas; relleno de lechos fluviales; construcción en pendientes cortando el perfil de las laderas; construcción de diques de tierra y enrocados para contención de aguas y "habilitación" de sectores de lechos fluviales mayores para construcción.

Estas tienen como objetivo: crear superficies de suelo urbanizable en áreas de alto valor del recurso, o en áreas de suelo escaso, o bien reducir los costos ocupando terrenos de mala calidad como suelo de fundación y, por lo tanto, de bajo precio, es decir, se busca maximizar la ganancia de algunas empresas sin medir el costo social.

En este caso, quisiéramos hacer referencia a dos situaciones que ilustran estas irracionalidades, por "alguien" permitidas, y que atentan contra la vida de los incautos compradores y contra sus bienes, resultado de toda una vida de sacrificio. 


\section{Emprendimientos "urbanísticos" en Iquique}

En Chile existe una serie de campos de dunas activas en el litoral, algunos de gran valor científico por constituir un laboratorio natural para el estudio de la dinámica de las arenas, formas de transporte y depositación, cementación y evolución pedagógica, mecanismos de avance sobre otros espacios comprometiendo diferentes usos del suelo y obras de infraestructura, y experimentación de medidas biológicas y estructurales para su posterior estabilización y posible aprovechamiento según las disponibilidades de humedad atmosférica.

Pues bien, sobre este tipo de unidades morfológicas producto de emprendimientos "urbanísticos" se están ejerciendo fuertes presiones y usos que rayan en la irracionalidad y generan en el corto y mediano plazo fuertes impactos socioeconómicos.

Tal es el caso de la población que en Iquique ha sido construida en los faldeos occidentales de una gran duna activa, el célebre Cerro Dragón. Cuáles son los problemas directos: Sedimento suelto en movimiento; alta deflación (extracción, transporte y retransporte de las arenas por el viento generando excavamiento de fundaciones, invasión de patios y casas, inestabilidad de pavimentos y postación); asentamientos de terreno por humedad (hundimientos por compactación o movilización del subsuelo arenoso asociados a filtraciones y riego); falla de cimientos, agrietamiento de murallas y descuadre de estructuras (marcos de puertas y ventanas).

Como consecuencia anexa, el ingreso de estos sedimentos finos al interior de las casas implica su incorporación a los aparatos eléctricos, a la cera de los pisos, a los alimentos y al aire que penetra en los pulmones, es decir, hay un deterioro manifiesto de la calidad de vida y de la salud de los moradores, especialmente en este caso en que la duna forma parte del patio trasero. 


\section{REVISTA DE URBANISMO \\ ISSN $0717-505$}

http://revistaurbanismo.uchile.cl

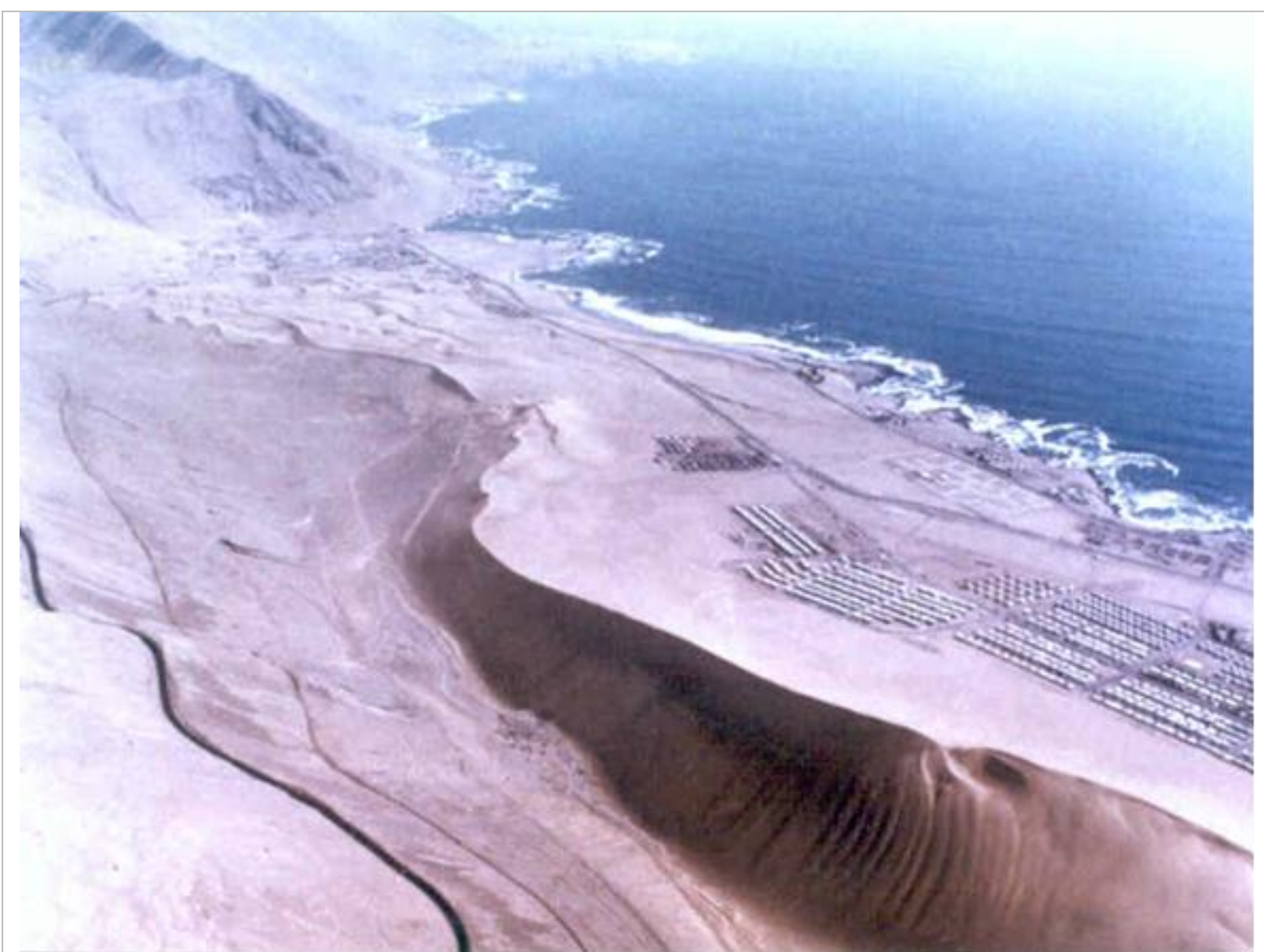

2.2.- Vista general de las poblaciones construidas en el pie occidental de la gran duna Cerro el Dragón, Iquique, sometidas a fuertes procesos deflacionarios (extracción y transporte de arenas por el viento) con graves problemas para las viviendas, redes de servicios básicos y personas. Foto: gentileza de Edgardo Gaete. 


\section{REVISTA DE URBANISMO \\ ISSN $0717-5051$}

La ciudad sin Urbanismo: instalación residencial junto a

http://revistaurbanismo.uchile.cl secciones andinas inestables, dunas activas, bancos de arena y rodados fluviales.

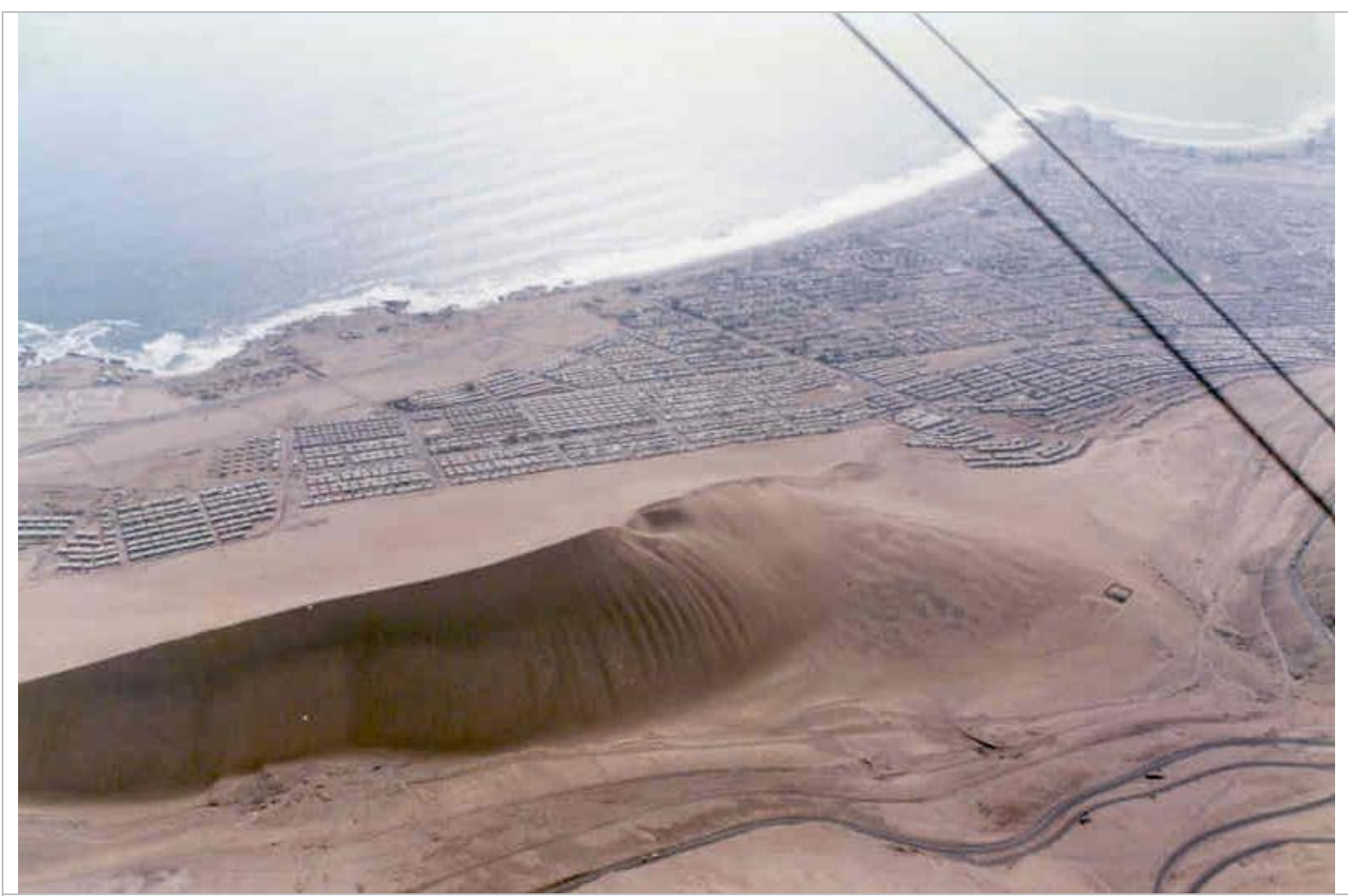

2.3.- Foto: gentileza de Edgardo Gaete.

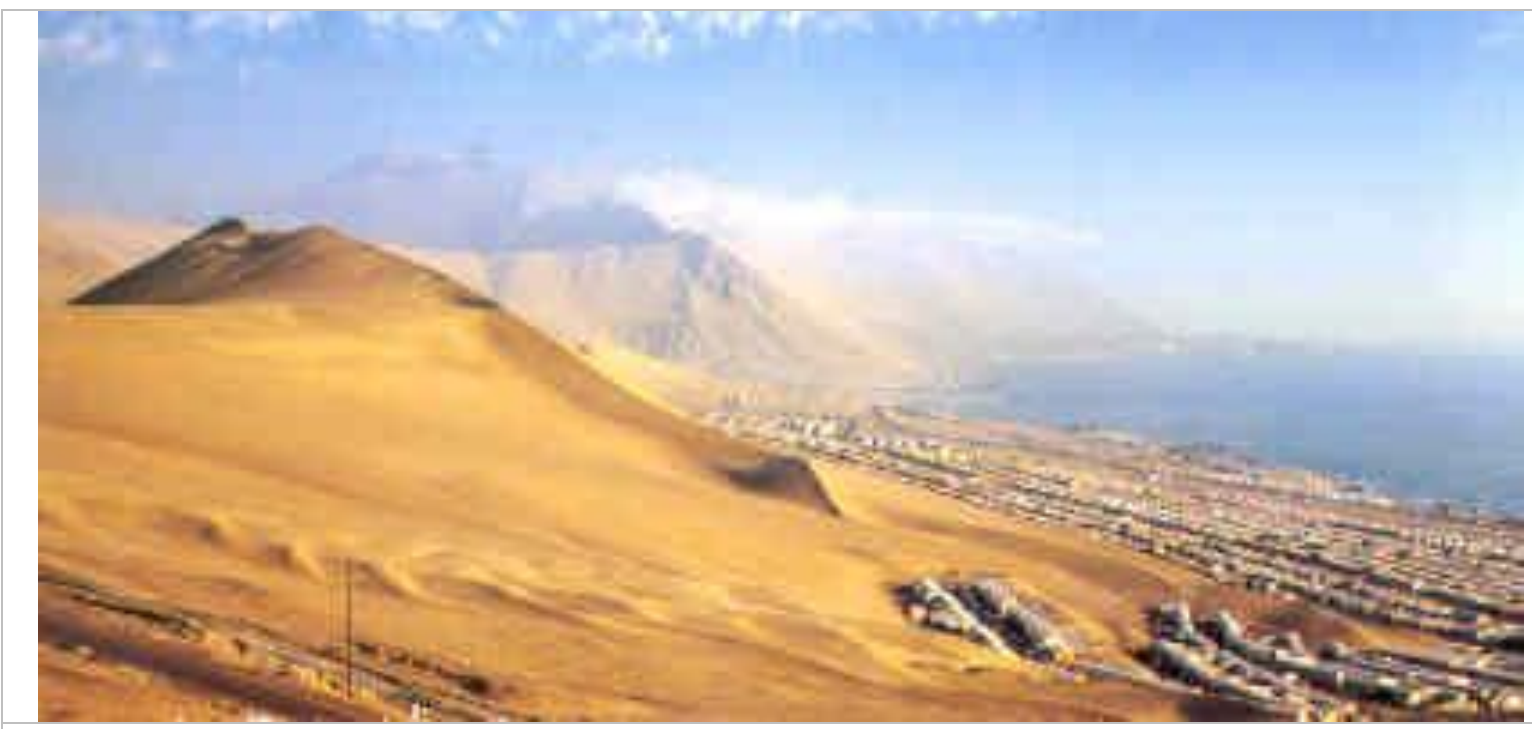

2.4.- Fte.: Sección de carta postal de Hispapel Ltda. Reproducción sin fines de lucro. 
http://revistaurbanismo.uchile.cl

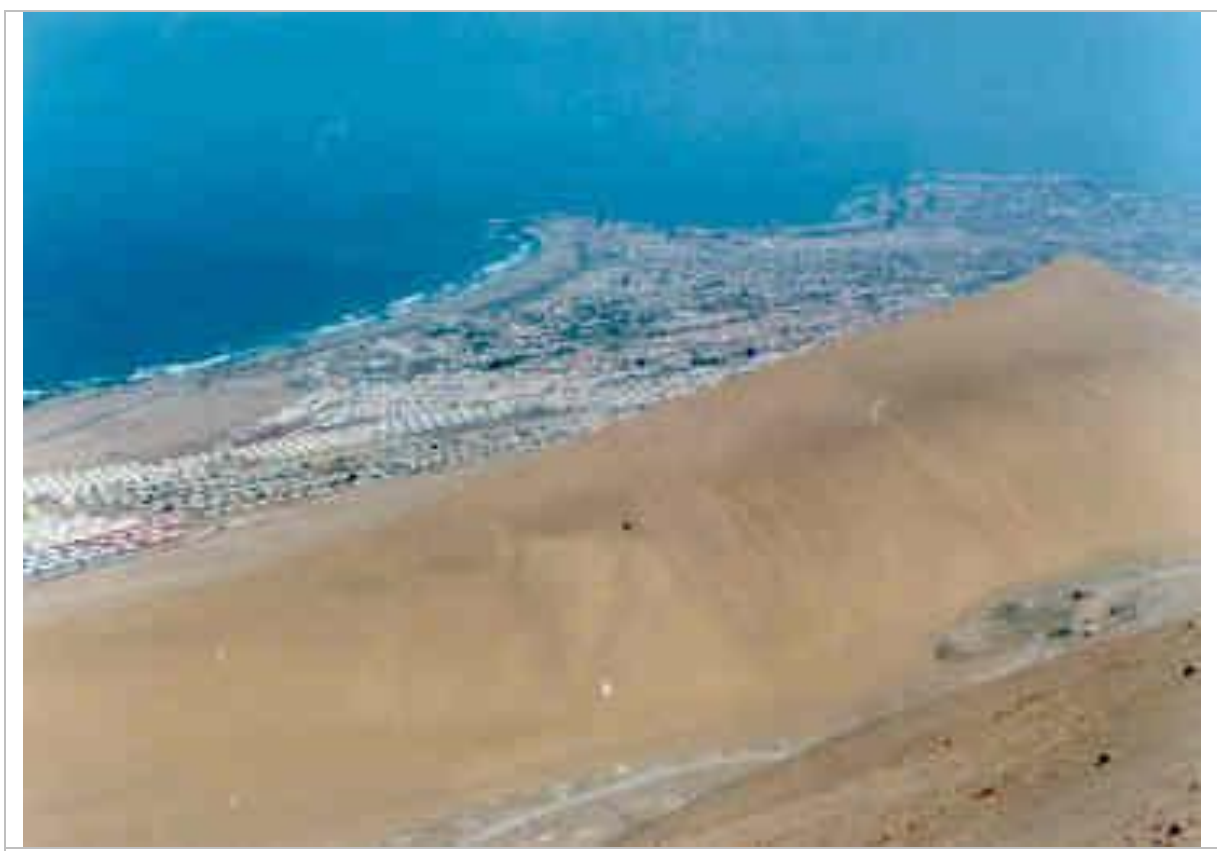

2.5.- Foto: gentileza de Edgardo Gaete.

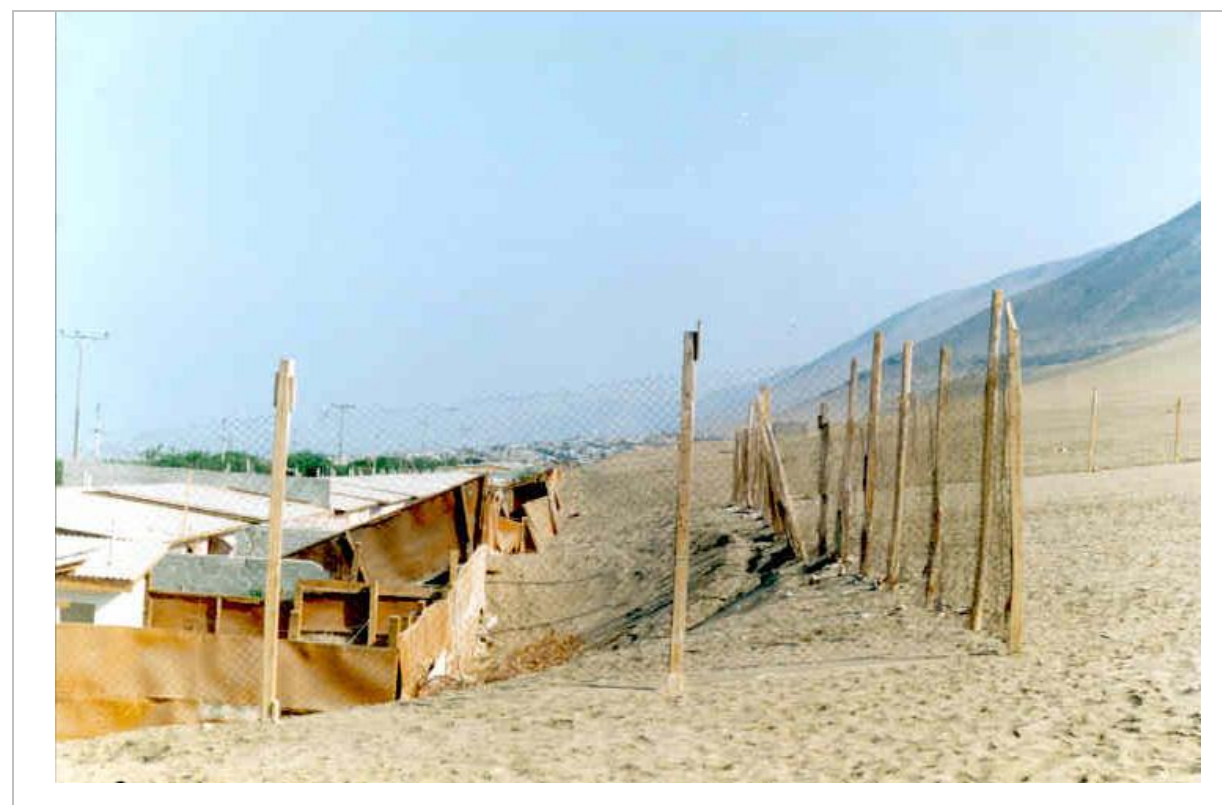

2.6.- Vista de la situación que afecta a las viviendas de la Población Huantajaya III, calle Los Algarrobos y calle 4, localizada al pie de la duna EI Dragón. Se aprecia el proceso de recuperación de perfiles de equilibrio después de corte de talud y precipitación de arenas sobre la construcción. Las arenas avanzan sobre los sitios, desestabilizan muros, invaden las casas por acción del viento. Foto: gentileza de Edgardo Gaete. 


\section{Construcción sobre bancos de arena y rodados fluviales de la terraza baja del río Cautín, Temuco}

A menos de un metro por sobre el nivel medio de las aguas encontramos población de viviendas económicas en Temuco, a orillas del río mencionado frente al aeropuerto. Se observa problemas por sedimento suelto, no consolidado, riesgo de inundaciones y erosión (pérdida de "suelos de fundación" y falla de las bases y la estructura), alta humedad del suelo por napa friática a pocos centímetros de la superficie (revenimiento de pisos y paredes, problemas de salud, deterioro de muebles, etc.). En realidad, no se sabe a ciencia cierta en qué aspectos estas viviendas son consecuentes con los logros contemporáneos para ofrecer marcos de vida satisfactorios.

Lo señalado, constatable a simple vista, deja al descubierto la existencia de graves problemas normativos y de incumplimiento de las disposiciones municipales. A este respecto, últimas modificaciones a las normas de construcción y urbanización son la causa de que se cometan irregularidades en la autorización de nuevas obras por las DOM. Esta afirmación dice relación con lo expresado en el Decreto Supremo No112, del 5 de Enero de 1993, el cual modifica la Ordenanza General de Urbanismo y Construcciones en lo referente a "Estudios de Riesgo", eliminando de su definición la expresión "de carácter obligatorio". De igual modo, la exigencia del "Informe de Riesgos" ya no queda supeditada a la definición del mismo, sino que a la aprobación de los permisos correspondientes, modificaciones que dejan abierta la posibilidad de manejo en cuanto a exigir estos estudios.

Las autoridades de turno, en algunos casos, suelen privilegiar el resolver problemas sociales con medidas basadas en visiones a corto plazo, dejando la atención de las consecuencias de estas, para la administración siguiente. En este sentido, la denuncia de la transgresión de las normas por obras construidas o en construcción, debiera poder ser llevada a cabo por organismos de base, como las Juntas de Vecinos, Consejos Comunales, o por simples particulares, debiendo, en estos casos, el Estado hacerse parte de los procesos, quizás incluso a través de la aplicación de una "ley de seguridad civil".

Pero, las situaciones y los riesgos señalados no son el único problema, ya que hay casos en que, "asumiendo su culpa" por las autorizaciones concedidas a empresas particulares, los municipios tratan de "tapar el problema" y acallar los reclamos mediante el desvío de fondos destinados a otras obras de beneficio social, para gastarlos en obras de defensa, las que no garantizan de modo adecuado la seguridad de la población. De hecho, la defensa construida a orillas del río Cautín no resistió y colapsó localmente el año 1993, frente a una situación de altas aguas medias -ni siquiera de una crecida de poca magnitud- hecho debido a que esta es autosoportante, tal como se aprecia en las fotos. 


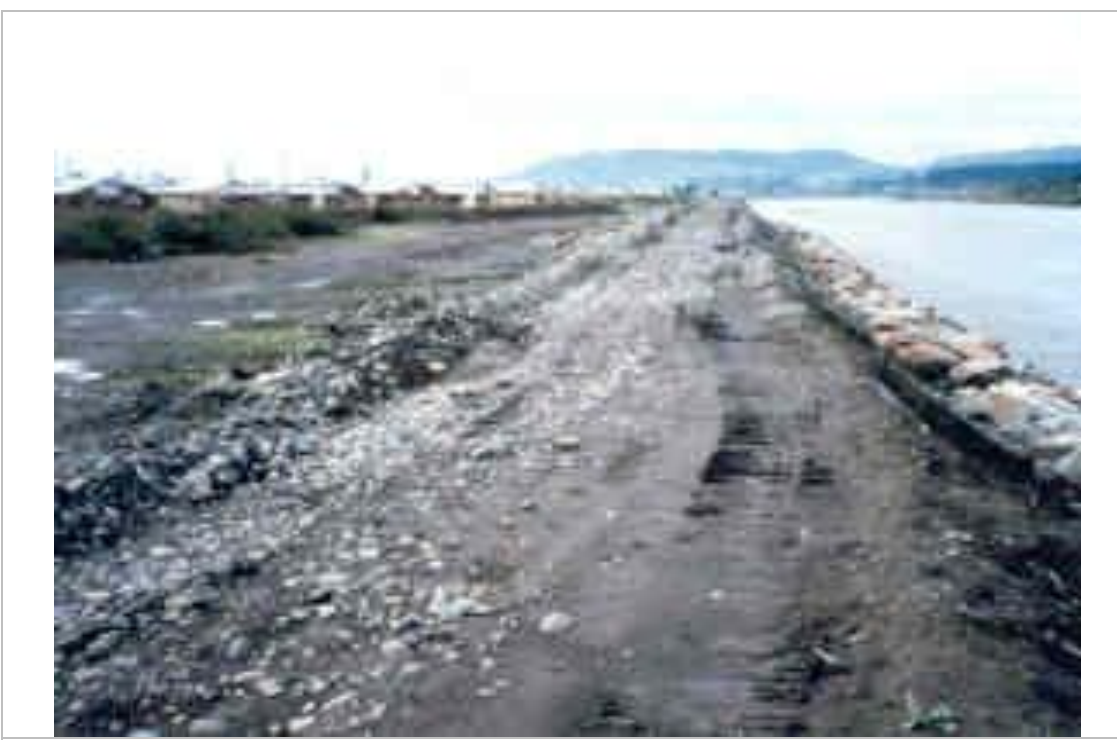

Fig.3.1.-Población construida en el lecho fluvial ocupado por el río Cautín en períodos de altas aguas medias. Se observa similar nivel entre el terreno (piso de las casas) y la superficie de las aguas. Defensa: muro de ripio y arena compactado, con malla plástica y enrocado en su frente expuesto.

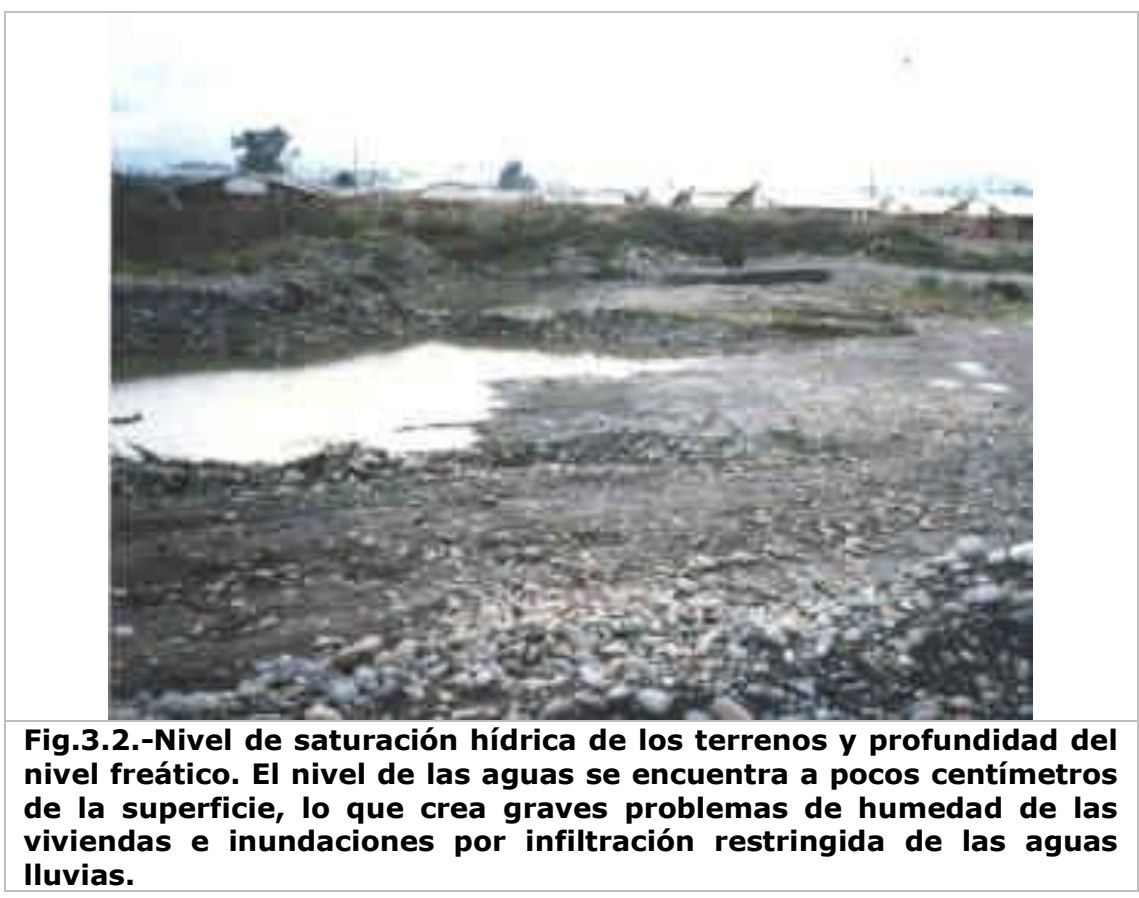


http://revistaurbanismo.uchile.cl

Por lo tanto, se crean situaciones de riesgo a sabiendas, y se incrementan significativamente las consecuencias y los costos en todo orden de cosas, como producto de la probable ocurrencia de un desastre por causas naturales en sectores que, sólo por sentido común y experiencia, se sabe que entrañan condiciones de vulnerabilidad, inestabilidad y peligro. La negligencia es hoy comparable a las fuerzas de la naturaleza en sus efectos.

En consecuencia, existe una serie de situaciones de riesgo que se debieran detectar y evaluar, a fin de tomar las medidas preventivas o correctivas a tiempo, cuyo costo debiera ser asumido por los verdaderos culpables, y no por la población directamente afectada, o el Estado.

Es evidente que es preferible prevenir antes que lamentar y asumir los costos, mucho mayores, de reparar lo reparable y lamentar lo irreparable (pérdida de vidas).

A este respecto, los organismos y las instancias existen, sólo se les debe dotar de mejores y más completas normativas e instrumentos de gestión, para corregir lo corregible, revocar las autorizaciones de lo inadecuado, y cautelar la sustentabilidad ambiental de los nuevos proyectos.

Paralelamente, se deben implementar medidas que efectivamente permitan reducir la energía de los procesos naturales, como los asociados a las cuencas hidrográficas, y mitigar sus efectos, mediante adecuados Planes de Manejo, lo cual compromete el espacio en sus tres dimensiones: natural, socioeconómica, y político-administrativa.

Finalmente, esto es un problema de conciencia y de ética, aspectos reñidos con los intereses económicos particulares, los que lamentablemente suelen imponerse sobre la lógica ambiental y la equidad social.

\section{Referencias}

Bibliografía para el Pto. 1.-

FERRANDO, F. \& MIKKAN, R., 2000. Estudio comparativo de asentamientos en areas de riesgo aluvional de Santiago (Ch.) y Mendoza (Ar.). Informe ejecutivo Proyecto IPGH 97-26. IPGH - OEA.

HAUSSER Y., A., 1985. "Flujos de barro en la zona preandina de la Región Metropolitana: Características, causas, efectos, riesgos y medidas preventivas". En Rev. Geológica 24:75-92.

LÓPEZ T., V., 1996. Evaluación físico ambiental de la cuenca de la Quebrada San Ramón. Memoria, Escuela de Geografía, F.A.U., Universidad de Chile.

PEÑA y VIDAL, 1993. "Estimación estadística de la línea de nieves durante los eventos de precipitación entre las latitudes 28 y 38 grados sur". En Actas XI Congreso Chileno de Ingeniería Hidráulica. 